\title{
Effects of manipulations to detect sliding hiatal hernia in dogs with brachycephalic airway obstructive syndrome
}

\author{
Olivier Broux DVM | Cécile Clercx DVM, PhD, Dipl. ECVIM-CA | \\ Anne-Laure Etienne DVM, PhD | Valeria Busoni DVM, PhD, Dipl. ECVDI | \\ Stéphanie Claeys DVM, PhD, Dipl. ECVS | Annick Hamaide DVM, PhD, Dipl. ECVS | \\ Frédéric Billen DVM, PhD, Dipl. ECVIM-CA
}

Department of Clinical Sciences, FARAH, Faculty of Veterinary Medicine,

University of Liege, Quartier Vallée 2,

Avenue de Cureghem 3 - B44, Liege,

Belgium

\section{Correspondence}

Olivier Broux, Small Animal Clinic, Faculty of Veterinary Medicine, Quartier Vallée 2, Avenue de Cureghem 3 - B44, 4000 Liège, Belgium.

Email: brouxolivier@hotmail.com

\begin{abstract}
Objective: To determine the influence of manipulations aimed at increasing the transdiaphragmatic pressure gradient on the gastro-esophageal junction (GEJ) of dogs with brachycephalic airway obstructive syndrome (BAOS), and to identify the manipulation that most improves the detection of GEJ abnormalities and sliding hiatal hernia (SHH) in dogs with BAOS.
\end{abstract}

Study design: In vivo experimental pilot study and prospective clinical study.

Animals: Five purpose-bred Beagles and 20 dogs diagnosed with BAOS.

Methods: Respiratory and digestive clinical signs as well as respiratory and GEJ abnormalities were scored. The presence of SHH was investigated using radiography and endoscopy in standard conditions. Endoscopic investigation was repeated after manipulations including manual pressure on the cranial abdomen (MP), Trendelenburg position $\left(30^{\circ}\right)$, or temporary complete endotracheal tube obstruction (ETO).

Results: No SHH was detected in any normal dog under any condition. Sixty-five percent of dogs with BAOS presented with digestive clinical signs, including vomiting and/or regurgitation. SHH was observed in only one dog via radiography and was not detected via endoscopy. Manipulations during endoscopy influenced GEJ abnormalities and allowed the detection of SHH in $2\left(30^{\circ}\right), 4$ (ETO), and 5 (MP) dogs, respectively. Digestive clinical signs correlated with GEJ abnormalities observed only in dogs with ETO $(P=.02)$.

Conclusion: Manipulations aimed at increasing the transdiaphragmatic pressure gradient during endoscopy in BAOS dogs allowed the detection of GEJ abnormalities and SHH that were not detected under standard conditions. Although MP allowed detection of SHH in more dogs than ETO, scores under MP did not correlate with digestive clinical signs. Therefore, ETO may be more accurate manipulation for the detection of GEJ abnormalities in BAOS dogs.

\section{1 | INTRODUCTION}

Sliding hiatal hernia (SHH), also known as axial or type I hiatal hernia, is a dynamic and intermittent cranial displacement of the gastro-esophageal junction (GEJ) into the thoracic cavity through the esophageal hiatus. Compared to
This study was presented at the resident forum of the 24th ECVS Annual Scientific Meeting. Berlin, Germany, July 2-4, 2015. 
other forms of hiatal hernias, $\mathrm{SHH}$ is by far the most frequently observed in $\operatorname{dog} s^{1-5}$ and has been described in French Bulldogs with brachycephalic airway obstructive syndrome (BAOS). ${ }^{6}$ SHH has been also occasionally observed in association with conditions such as chronic diaphragmatic hernia, ${ }^{7,8}$ tetanus, ${ }^{9}$ or laryngeal paralysis. ${ }^{10}$ Several theories have been proposed to explain the etiology of SHH. An increase in transdiaphragmatic pressure gradient (ie, the difference between intra-abdominal and intrathoracic pressures) could induce an axial separation of the lower esophageal sphincter from extrinsic factors, such as the diaphragmatic crural muscle, thereby weakening the GEJ barrier pressure and allowing gastro-esophageal reflux. ${ }^{7,11-16}$ Subsequent esophagitis may further decrease lower esophageal sphincter pressures, aggravating reflux and resulting in a selfperpetuating cycle. ${ }^{17-19} \mathrm{~A}$ second theory is that a shortening of the esophagus caused by a vagal stimulation pulls the GEJ through the esophageal hiatus. This shortening could be physiological during swallowing. ${ }^{20,21}$ Finally, SHH could result from a congenital or acquired abnormally wide hiatus. ${ }^{14}$

Clinical evaluation of BAOS patients should include at least neck and thoracic radiographs, as well as endoscopic examination of the upper airways. ${ }^{16}$ However, no gold standard exists for the investigation of the upper gastrointestinal tract. The diagnosis of SHH in human patients is established based on thoracic and contrast radiographs, fluoroscopy, endoscopy, and manometry. ${ }^{22}$ In veterinary medicine, the prevalence of canine SHH with BAOS has only been investigated using standard endoscopy. While upper digestive clinical signs were observed in $74 \%$ and $93 \%$ of those dogs, SHH was reported in only $4.1 \%$ and $3.3 \%$ of those dogs, respectively. ${ }^{6,23}$ The absence of upper respiratory obstruction and inspiratory efforts during standard endoscopic procedures could explain the low prevalence of SHH observed in these studies. Compressing the abdomen during radiography in patients with suspected SHH has been proposed to force part of the stomach into the thorax and increase the likelihood of diagnostic images. ${ }^{24}$ To the authors' knowledge, no prospective study has determined the influence of an increased transdiaphragmatic pressure gradient on the GEJ, including the occurrence of SHH, in dogs with BAOS.

The aims of the study were to identify manipulations that would best improve the detection of GEJ abnormalities and SHH in dogs with BAOS. We hypothesized that artificially increasing the pressure gradient between the abdominal and thoracic cavities during diagnostic procedures, by either increasing intra-abdominal pressure or decreasing intrathoracic pressure, could improve the detection of GEJ abnormalities and SHH in dogs with BAOS.

\section{2 | MATERIALS AND METHODS}

The study protocol was approved by the Ethical Commission of Animal use of the institution (number 1425). Five healthy purpose-bred Beagles were included in a pilot study to evaluate the safety and feasibility of the protocol. All examinations on BAOS dogs were performed with informed owner's consent.

\section{1 | Study population and standard diagnostic procedures}

Dogs presented for respiratory clinical signs compatible with BAOS, with or without digestive clinical signs, and without concurrent diseases were prospectively recruited between June and October 2014. Breed, age, sex, body weight, body condition score, and history were recorded. A complete physical examination was performed. Three view thoracic radiographs (ventro-dorsal, right, and left lateral) (Digivex FP Medex 15 kW; 833539G015, Medex Digivex, Loncin, Belgium) were obtained under mild sedation using medetomidine $(5-20 \mu \mathrm{g} / \mathrm{kg}$ IV) and/or butorphanol $(0.1-0.2 \mathrm{mg} / \mathrm{kg}$ IV). Images were reviewed by one board certified radiologist to investigate the presence of tracheal hypoplasia, aspiration bronchopneumonia, and SHH. Routine endoscopic investigation (Fujinon gastroscope, $5.2 \mathrm{~mm}, 100 \mathrm{~cm}$; EG-470N5, Fujinon, Saitama City, Japan) of the upper respiratory tract and of the upper gastrointestinal tract, including the GEJ, was initially performed in left lateral recumbency under general anesthesia. Medetomidine (5-20 $\mu \mathrm{g} / \mathrm{kg}$ IV) and/or butorphanol $(0.1-0.2 \mathrm{mg} / \mathrm{kg} \mathrm{IV})$ were used for premedication, followed by propofol $(1-5 \mathrm{mg} / \mathrm{kg} \mathrm{IV})$ for induction and isoflurane (1.5\%-2\% end-tidal measurement) in 100\% oxygen for maintenance. In order to investigate the repeatability of the scoring system, all endoscopic videos were reviewed blindly by 3 veterinarians ( 1 board certified internist, 1 surgery resident, and 1 intern) and assessed twice by each reviewer.

\section{2 | Manipulations aimed at increasing the transdiaphragmatic pressure gradient}

Manipulations were performed only during esophagoscopy to avoid excessive stress or prolonged anesthesia during radiographs. To assess the effect of an increased intra-abdominal pressure on the GEJ, endoscopic evaluation was repeated during manual pressure on the cranial abdomen with the fist (MP) (Figure 1) as previously described, ${ }^{24}$ and during body angulation at $30^{\circ}$ Trendelenburg position in left lateral recumbency $\left(30^{\circ}\right.$ ) (Figure 2). To assess the effect of a decreased intra-thoracic pressure on the GEJ, the endotracheal tube obstruction (ETO) was completely obstructed during 3 spontaneous inspirations or a maximum of 30 seconds, in order to reproduce deep inspiratory efforts during episodes of upper airways obstruction (Figure 3). Manipulations were performed after the standard evaluation in a random order drawn between the 6 different possibilities ( $30^{\circ}$-MP-ETO; $30^{\circ}$-ETO-MP; 


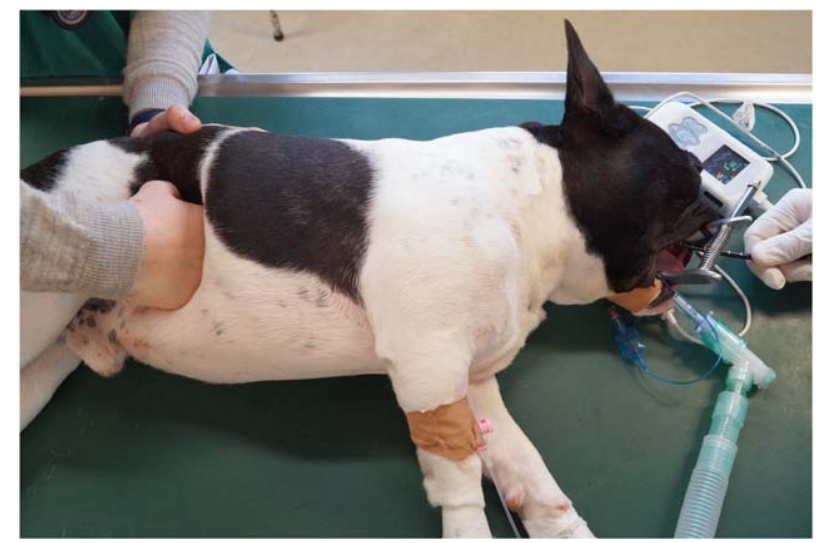

FIGURE 1 Manual pressure is applied with the first to the cranial abdomen (MP) in a French Bulldog during esophageal endoscopy

ETO-MP-30² ETO-30-MP; MP-ETO-30 ${ }^{\circ}$ MP-30-ETO), ensuring that each order was used at the same frequency (3-4 times each). A board certified anesthetist monitored each dog closely (heart and respiratory rates, oxygen saturation, capnography, and blood pressure) during endoscopy.

\section{3 | Scoring}

Respiratory and digestive clinical scores (each ranging from 0 to 4) (Tables 1 and 2), as well as a respiratory abnormalities score (ranging from 0 to 9) and a GEJ abnormalities score (ranging from 0 to 7 ) (Table 3 ) were assigned. The clinical grading systems were adapted from Poncet and collaborators ${ }^{6}$ and were based on history of clinical signs. The respiratory clinical score was based on the frequency of snoring, inspiratory efforts, exercise intolerance, and cyanosis or syncope. On the basis of the frequency of each respiratory sign, a global classification of 5 grades was obtained: absent (grade 0), minimal (grade 1), moderate (grade 2), severe (grade 3 ), or extremely severe (grade 4) (Table 1). The respiratory clinical score is determined by the highest grade. Since differentiation between regurgitation and

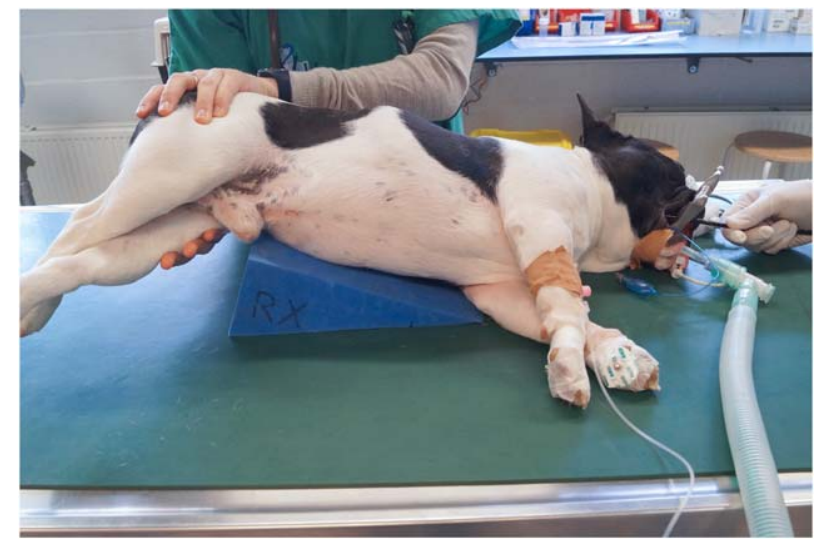

F IGURE 2 Body angulation at $30^{\circ}$ Trendelenburg position in left lateral recumbency $\left(30^{\circ}\right)$ in a French Bulldog during esophageal endoscopy

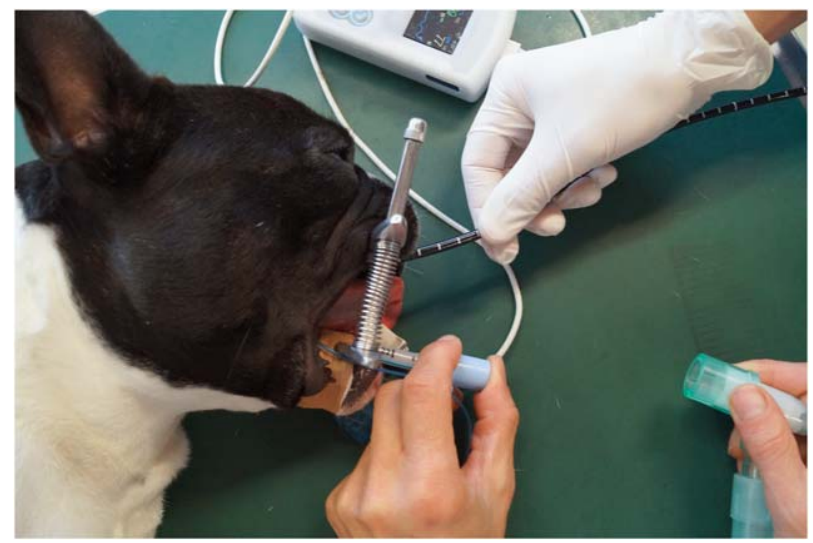

FIGURE 3 Temporary complete endotracheal tube obstruction in a French Bulldog during esophageal endoscopy

vomiting can be difficult for owners, the digestive clinical score was based on the frequency of both regurgitations and/or vomiting, and a global classification of 5 grades was obtained: from absent (grade 0) to frequent (grade 4) (Table 2). The respiratory abnormalities score was adapted from Bernaerts and collaborators $^{25}$ and was based on clinical examination (stenotic nares), radiographic (tracheal hypoplasia), and endoscopic findings (soft palate elongation and laryngeal collapse) (Table 3). We established a scoring system for the GEJ abnormalities based on endoscopic findings (cardia opening, gastro-esophageal reflux, and GEJ sliding) (Table 3). GEJ sliding was defined as the evaluation of the cranial displacement of the GEJ into the thoracic cavity (Figure 4); the presence of bulging and eversion of the gastric mucosa for more than $2 \mathrm{~cm}$ cranial to the esophageal hiatus was considered as SHH (Figure 5). The $2 \mathrm{~cm}$ were measured with an intraesophageal sizing catheter (Infiniti Medical, London, UK). The GEJ abnormalities score was obtained in standard conditions as well as during MP, $30^{\circ}$, and ETO. For each manipulation, the final GEJ abnormalities score was determined as the mean of the 6 scores established by 3 independent readers on 2 separate occasions.

\subsection{Statistical analysis}

Statistical analysis was performed with $\mathrm{R}$ program (Version 3.1.3). Univariate analysis (Shapiro-Wilk normality tests) was used to assess normal distribution of the various dependent variables. To analyze the influence of certain factors, such as the manipulations, and of covariables on the endoscopic scores, a generalized linear model with a distribution of residues following a law of Poisson was used to answer the nonnormality of the observed clinical scores. We also used non parametric ANOVA based on the data rank, especially to test the repeatability of the data between readings and readers of the endoscopies. For all analysis, a threshold of 5\% meaning $(P<.05)$ was considered statistically significant. Kendall correlation tests were performed between each pair of scoring parameters. 
TABLE 1 Respiratory clinical score grading system ${ }^{\mathrm{a}}$

\begin{tabular}{|c|c|c|c|c|c|}
\hline Respiratory clinical signs & Absent & $\begin{array}{l}\text { Occasionally } \\
(\leq \mathbf{1} / \mathbf{m o})\end{array}$ & $\begin{array}{l}\text { Regularly } \\
(\leq 1 / w k)\end{array}$ & $\begin{array}{l}\text { Daily } \\
(\leq 1 / d)\end{array}$ & $\begin{array}{l}\text { Often } \\
(>1 / d)\end{array}$ \\
\hline Snoring & 0 & 0 & 0 & 1 & 2 \\
\hline Inspiratory efforts & 0 & 1 & 2 & 3 & 4 \\
\hline Exercise intolerance & 0 & 1 & 2 & 3 & 4 \\
\hline Cyanosis/syncope & 0 & 3 & 4 & 4 & 4 \\
\hline
\end{tabular}

${ }^{\mathrm{a}}$ The respiratory clinical score was based on the frequency of the different clinical signs. The highest grade determines the score (ranging from 0 to 4 ) (adapted from Poncet et al, 2005).

\section{3 | RESULTS}

\section{1 | Pilot study}

Five healthy adult purpose-bred Beagles, 4 females and 1 male, with a mean age of 27 months, a mean body weight of $12.5 \mathrm{~kg}$ (range, 11.0-13.2), and a median body condition score of 5/9 (range, 4-5) were used for the pilot study. The digestive clinical scores, respiratory clinical scores, and respiratory abnormalities scores were 0 for all Beagles. Neither standard radiographs, nor any manipulation during endoscopy were consistent with SHH. The median GEJ abnormalities score (from 0 to 7 ) was 0 (standard), 0 (30 $), 0$ (ETO), and 1 (MP), respectively. Only MP induced opening of the cardia in 2 Beagles. Eversion of the gastric mucosa and gastro-esophageal reflux were never observed. All dogs recovered from anesthesia without complication.

\section{2 | Study population}

Twenty dogs with BAOS met the inclusion criteria. There were 15 males and 5 females, 6 French Bulldogs, 6 English Bulldogs, 3 Pugs, 2 Cavalier King Charles Spaniel, and 1 of Bullmastiff, Maltese Bichon, and Shih Tzu. Mean age at presentation was 34 months (range, 6-98). A mean body weight of $15.2 \mathrm{~kg}$ (range, 5.7-40.5) and a median body condition score of 6/9 (range, 2-8) were observed. All dogs presented with respiratory signs, with a median respiratory clinical score of 2.0 (range, 1-4, Table 4). Only $65 \%$ of the dogs presented with digestive clinical signs, with a median digestive clinical score of 2.0 (range, 0-4). The median respiratory abnormalities score was 5.0 (range, 3-9). Tracheal hypoplasia was noted on radiographs of 5 dogs, without aspiration bronchopneumonia. SHH was detected on radiographs in one dog. The median GEJ abnormalities score was 2.0 (Standard), $2.0\left(30^{\circ}\right), 2.0$ (ETO), and 3.0 (MP). SHH was observed in 0 (Standard), $2\left(30^{\circ}\right), 4$ (ETO), and 5 (MP) dogs, respectively. The GEJ abnormalities observed during endoscopy are summarized in Table 5. Compared to standard conditions, the mean GEJ abnormalities score (Table 6) increased during the 3 manipulations but the median did not differ $(P>.05)$. There was no difference $(P<.05)$ in GEJ abnormalities score and detection of SHH (during standard conditions and the 3 manipulations) between the 2 readings and the 3 readers (Table 7).

\section{3 | Correlations between scores}

Correlations were detected between the 2 clinical scores (respiratory and digestive) $(z=2.77 ; P=.003$; tau $=0.54)$, the 2 respiratory scores (clinical and abnormalities) $(z=3.94 ; P=.00004 ;$ tau $=0.76)$, and between digestive clinical score and respiratory abnormalities score $(z=3.80$; $P=.00007 ; \mathrm{tau}=0.71)$. The GEJ abnormalities scores correlated with the digestive clinical scores $(z=2.00 ; P=.02$; $\operatorname{tau}=0.36)$ and with the respiratory abnormalities scores $(z=1.90 ; P=.03 ;$ tau $=0.33)$ during ETO only (Figure 6$).$

\section{4 | Morbidity and recovery}

Manipulations did not increase the morbidity of the endoscopic examinations. Heart and respiratory rates, oxygen saturation, and blood pressure remained stable throughout all

TABLE 2 Digestive clinical score grading system ${ }^{\mathrm{a}}$

$\begin{array}{lllll}\text { Digestive clinical signs } & \text { Absent } & \begin{array}{l}\text { Occasionally } \\ (\leq \mathbf{1} / \mathbf{m o})\end{array} & \begin{array}{l}\text { Regularly } \\ (\leq \mathbf{1} / \mathbf{w k})\end{array} & \begin{array}{l}\text { Daily } \\ (\leq \mathbf{1} / \mathbf{d})\end{array} \\ \text { Regurgitation or vomiting } & 0 & 1 & 2 & 3 \\ (>\mathbf{1} / \mathbf{d})\end{array}$

${ }^{\text {a }}$ The digestive clinical score was based on the frequency of vomiting or regurgitation (ranging from 0 to 4) (adapted from Poncet et al, 2005). 
TABLE 3 Respiratory abnormalities score and GEJ abnormalities score grading systems ${ }^{\mathrm{a}}$

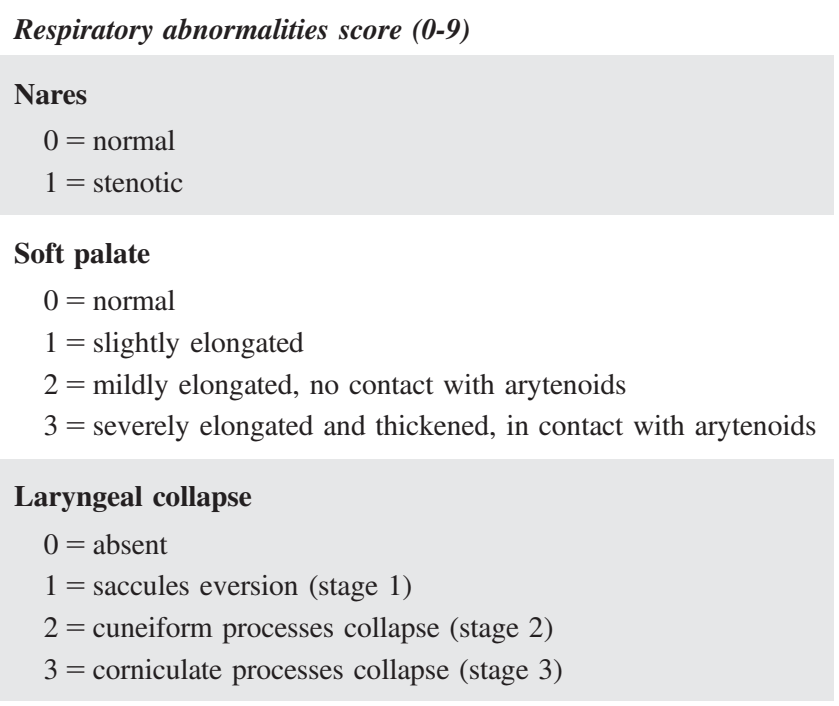

\section{Tracheal hypoplasia}

$$
\begin{aligned}
& 0=\text { absent } \\
& 1=\text { mild } \\
& 2=\text { severe }
\end{aligned}
$$

GEJ abnormalities score (0-7)

$$
\begin{aligned}
& \text { Cardia } \\
& \begin{aligned}
0=\text { closed } \\
1=\text { opens } \\
2=\text { gaping }
\end{aligned}
\end{aligned}
$$

$$
\begin{aligned}
& 0=\text { absent } \\
& 1=\text { intermittent } \\
& 2=\text { permanent }
\end{aligned}
$$$$
\text { Gastro-esophageal reflux }
$$

\section{GEJ sliding}

$0=$ absent

$1=$ bulging and partial circumferential gastric mucosa eversion

$2=$ bulging and complete circumferential gastric mucosa eversion $3=\mathrm{SHH}$

${ }^{\text {a }}$ The respiratory abnormalities score was based on physical examination, thoracic radiographs, and laryngoscopy. The sum of the grades determines the score (ranging from 0 to 9) (adapted from Bernaerts et al, 2010). The GEJ abnormalities score was based on abnormalities of the GEJ observed during endoscopy of the esophagus. The sum of the grades determines the score (ranging from 0 to 7). Abbreviations: GEJ, gastro-esophageal junction; SHH, sliding hiatal hernia.

manipulations. All dogs recovered from anesthesia without complication.

\section{4 | DISCUSSION}

This is the first prospective study evaluating the effects of transdiaphragmatic pressure gradients on the GEJ during

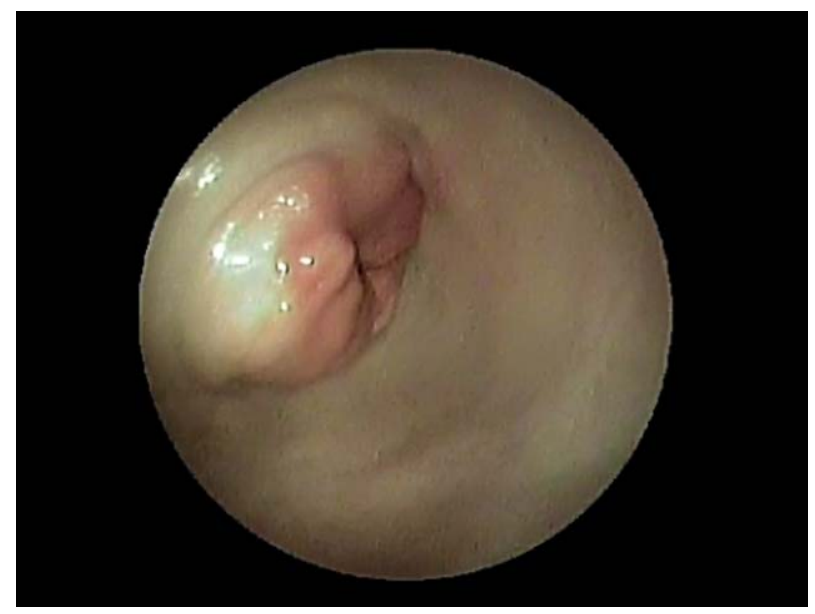

FIGURE 4 Bulging and eversion of the gastric mucosa in a dog with brachycephalic airway obstructive syndrome observed during esophageal endoscopy

endoscopy of dogs with BAOS. Although $65 \%$ of dogs presented with digestive clinical signs, only one SHH was diagnosed with standard radiography and none with standard endoscopy. Increasing the transdiaphragmatic pressure gradient by manipulations during endoscopy allowed the detection of additional abnormalities of the GEJ compared to standard conditions. This study supports our hypothesis that an increase in transdiaphragmatic pressure gradient impacts the GEJ and that GEJ abnormalities, including SHH, are underdiagnosed using standard diagnostic procedures in dogs with BAOS. According to the results of the present study, ETO allows the detection of GEJ abnormalities that could be associated with digestive clinical signs.

Thoracic radiography allowed detection of SHH in only one dog with BAOS, and therefore appeared unreliable as a diagnostic tool for this condition. A radiograph provides a static image, and may not be adequate to detect dynamic abnormalities. Furthermore, a study on adult human population of North America reported a prevalence of type I hiatal

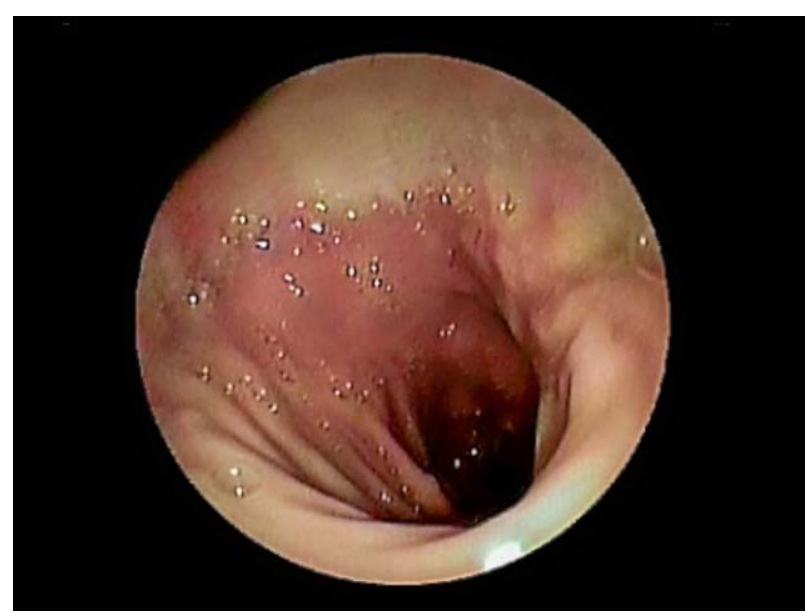

FIG URE 5 Sliding hiatal hernia in a dog with brachycephalic airway obstructive syndrome observed during esophageal endoscopy 
TABLE 4 Summary of the clinical signs and the respiratory abnormalities $^{\mathrm{a}}$

\begin{tabular}{|ll|}
\hline Clinical signs and abnormalities & $\begin{array}{l}\text { Number of } \\
\text { dogs } \mathbf{( 0 - 2 0})\end{array}$ \\
\hline Digestive clinical signs & 13 \\
Regurgitation & 1 \\
Vomiting & 3 \\
Both & 9 \\
\hline Respiratory clinical signs & 20 \\
Snoring & 19 \\
Inspiratory efforts & 7 \\
Exercise intolerance & 11 \\
Cyanosis/syncope & 6 \\
Respiratory abnormalities & 20 \\
Stenotic nares & 14 \\
Soft palate elongation & 20 \\
Laryngeal collapse & 10 \\
Tracheal hypoplasia & 5 \\
\hline
\end{tabular}

${ }^{\mathrm{a}}$ Number of dogs (from 0 to 20 ) presented with each clinical sign and diagnosed with each respiratory abnormality.

hernia varying from $10 \%$ to $80 \%$, because of the subjectivity of the radiographic criteria. ${ }^{26}$ Increasing the transdiaphragmatic pressure gradient by manipulations during thoracic radiographs could potentially help detect SHH. However, manipulations were not performed during thoracic radiographs in this study to avoid excessive stress if performed without anesthesia, or to avoid prolonged anesthesia. In addition, generating excessive transdiaphragmatic pressure gradients by manipulations could potentially induce false positive gastro-esophageal reflux and modify the results of subsequent standard endoscopy.

In contrast to radiography, endoscopy enables dynamic examination and should improve the detection of SHH. We established a scoring system for endoscopic GEJ abnormalities, in order to expand our assessment to other abnormalities, such as minor sliding or gastro-esophageal reflux. This scoring system was considered reliable, repeatable, and independent of the reader's expertise. We decided to exclude esophagitis (detected in 11 dogs) from the scoring system to limit our assessment to the dynamic aspect of GEJ abnormalities. The prevalence of SHH in dogs with BAOS seems low compared to the high prevalence of digestive clinical signs in the population studied here, as well as in previous publications. ${ }^{6,23}$ Intubation of anesthetized dogs decreases the risk of respiratory obstruction, and may mask GEJ abnormalities and SHH. Body position and anesthetic agents could also influence the detection of GEJ abnormalities. Indeed, body position and some anaesthetic agents, such as acepromazine, have been found to reduce the pressure within the lower esophageal sphincter and affect the GEJ function. ${ }^{27,28}$ Endoscopy was in left lateral recumbency in
TABLE 5 Summary of the abnormalities of the GEJ in standard conditions and during the 3 manipulations

\begin{tabular}{lllll} 
& \multicolumn{4}{l}{ Number of dogs (0-20) } \\
\cline { 2 - 5 } GEJ abnormalities & STD & $\mathbf{3 0}$ & ETO & MP \\
Cardia & & & & \\
Closed & 11 & 13 & 11 & 6 \\
Opened & 6 & 4 & 2 & 8 \\
Gaping & 3 & 3 & 7 & 6 \\
\hline GER & & & & \\
Absent & 20 & 15 & 16 & 11 \\
Intermittent & 0 & 5 & 4 & 9 \\
Permanent & 0 & 0 & 0 & 0 \\
Sliding & & & & \\
Absent & 5 & 3 & 4 & 0 \\
Bulging & 8 & 7 & 7 & 7 \\
Eversion & 7 & 8 & 5 & 8 \\
SHH & 0 & 2 & 4 & 5 \\
\hline
\end{tabular}

Abbreviations: $30^{\circ}, 30^{\circ}$ Trendelenburg position in left lateral recumbency; ETO, temporary complete endotracheal tube obstruction; GEJ, gastro-esophageal junction; GER, gastro-esophageal reflux; MP, manual pressure, with the fist, on the cranial abdomen; SHH, sliding hiatal hernia; STD, standard conditions.

our study, because this position has been reported to have the least effects on GEJ pressure. ${ }^{22}$ Since endoscopic evaluation of the GEJ requires anesthesia, we prioritized the selection of a safe protocol for dogs with BAOS, over a protocol that would minimize interferences with the GEJ function.

An increased transdiaphragmatic pressure gradient has been proposed as a causative factor for SHH in dogs with BAOS. Indeed, aerophagia and delayed gastric emptying in diseased dogs could increase the intra-abdominal pressure, ${ }^{6}$ which may induce a cranial displacement of the GEJ. On the other hand, increased inspiratory efforts in the presence of upper airways obstruction are thought to decrease the intrathoracic pressure, therefore inducing $\mathrm{SHH}^{6,14,16,24}$ To the authors' knowledge, this is the first study showing that increasing the transdiaphragmatic pressure gradient has an impact on the GEJ in dogs with BAOS. Each

TABLE 6 Summary of the GEJ abnormalities scores in standard conditions and during the 3 manipulations

\begin{tabular}{lllll|} 
GEJAS (0-7) & STD & $\mathbf{3 0}^{\circ}$ & ETO & MP \\
\hline Mean & 1.7 & 2.0 & 2.4 & 3.2 \\
\hline Median & 2.0 & 2.0 & 2.0 & 3.0 \\
\hline Range & $0-3.3$ & $0-4.8$ & $0-5.7$ & $0-4.7$ \\
\hline
\end{tabular}

Abbreviations: $30^{\circ}, 30^{\circ}$ Trendelenburg position in left lateral recumbency; ETO, temporary complete endotracheal tube obstruction; GEJAS, gastroesophageal abnormalities score; MP, manual pressure, with the fist, on the cranial abdomen; STD, standard conditions. 
TABLE 7 Nonparametric ANOVA results for repeatability of the GEJ abnormalities score and the detection of SHH between the 2 readings and the 3 readers ${ }^{\mathrm{a}}$

\begin{tabular}{llllll} 
& \multicolumn{5}{c}{ Readers } \\
\cline { 3 - 6 } $\begin{array}{l}\text { Repeatability } \\
\text { (P-values) }\end{array}$ & $\begin{array}{l}\text { Readings } \\
\mathbf{1} \text { and } \mathbf{2}\end{array}$ & $\mathbf{1}$ and $\mathbf{2}$ & $\mathbf{1}$ and 3 & $\mathbf{2}$ and 3 & $\begin{array}{l}\mathbf{1 , 2} \\
\text { and 3 }\end{array}$ \\
GEJAS & & & & & \\
STD & 0.71 & 0.88 & 0.66 & 0.92 & 0.68 \\
$30^{\circ}$ & 0.49 & 0.94 & 0.93 & 1.00 & 0.92 \\
ETO & 0.35 & 1.00 & 0.97 & 0.96 & 0.96 \\
MP & 0.43 & 0.69 & 0.79 & 0.98 & 0.70 \\
SHH & & & & & \\
STD & 0.89 & 0.86 & 0.86 & 1.00 & 0.84 \\
$30^{\circ}$ & 0.90 & 0.94 & 0.99 & 0.99 & 0.94 \\
ETO & 0.44 & 0.95 & 0.95 & 1.00 & 0.94 \\
MP & 0.71 & 0.89 & 0.82 & 0.99 & 0.83 \\
\hline
\end{tabular}

${ }^{\text {a }} P$-value $>.05$ lead to accept the hypothesis of identical scores between the readers and the readings.

Abbreviations: $30^{\circ}, 30^{\circ}$ Trendelenburg position in left lateral recumbency; ETO, temporary complete endotracheal tube obstruction; GEJAS, GEJ abnormalities score; MP, manual pressure, with the fist, on the cranial abdomen; SHH, sliding hiatal hernia; STD, standard conditions.

manipulation increased the GEJ abnormalities score and the number of SHH detected in comparison to standard endoscopy. Although we assume that the manipulations did modify the transdiaphragmatic pressure gradient, we did not effectively measure pressure variations. Further studies using esophageal manometry or dual thoracoabdominal respiratory inductance plethysmography, as recommended by the American Academy of Sleep Medicine, ${ }^{29}$ are necessary to quantify changes in transdiaphragmatic pressure gradients.

The prevalence of digestive clinical signs (65\%) in dogs enrolled in our study compares favorably to that reported by Poncet and collaborators ${ }^{6,23}$ (74\% and $93 \%$ of dogs, respectively). We confirmed the correlation between the respiratory and digestive clinical signs, previously reported in these studies. Endotracheal intubation was the only manipulation that revealed a correlation between the digestive clinical and the GEJ abnormalities scores in our study. ETO is a simple method to decrease the intrathoracic pressure. Based on our results, this technique may best reproduce the dynamics of the GEJ (SHH and other GEJ abnormalities) in awake dogs with BAOS, during episodes of upper airway obstruction. By contrast, the 2 other manipulations (MP and $30^{\circ}$ ), designed to increase intra-abdominal pressure, did not correlate the digestive clinical and the GEJ abnormalities scores, and were therefore considered less adequate to reveal GEJ abnormalities in dogs with BAOS. Furthermore, MP could overestimate while standard conditions and $30^{\circ}$ could underestimate GEJ abnormalities and SHH in dogs with BAOS.
Indeed, standard conditions and $30^{\circ}$ detected less SHH than ETO, while MP detected more SHH and more severe GEJ abnormalities compared to ETO. Alternatively, the low number of dogs in this study may have affected our ability to detect correlations between the digestive clinical and the GEJ abnormalities scores with the other manipulations. Further studies are needed in a larger population of BAOS dogs to confirm this finding. Our study focused on dynamic abnormalities of the GEJ, but did not evaluate other abnormalities that could lead to digestive clinical signs in dogs with BAOS. We only included dogs that were free of other concurrent diseases, and performed a complete endoscopic investigation of the upper digestive system in all dogs. No mucosal abnormalities, pyloric stenosis, pyloric atony, nor duodenogastric reflux was observed. We could have evaluated esophageal deviation and the presence of gastritis as Poncet and collaborators. ${ }^{6}$ However, the correlation observed between digestive clinical score and GEJ abnormalities score during ETO in this study indicates that the dynamic abnormalities of the GEJ may be implicated in the pathogenesis of the digestive clinical signs in dogs with BAOS. Conversely, this correlation could explain the improvement of digestive clinical signs after surgical correction of obstructing upper airways, and warrant further exploration in large population of dogs with BAOS monitored postoperatively. ${ }^{6}$ In contrast to other studies, ${ }^{6,23,30,31}$ we were unable to detect a breed, gender, or body condition score predisposition for $\mathrm{SHH}$, potentially due to the lower number of patients.

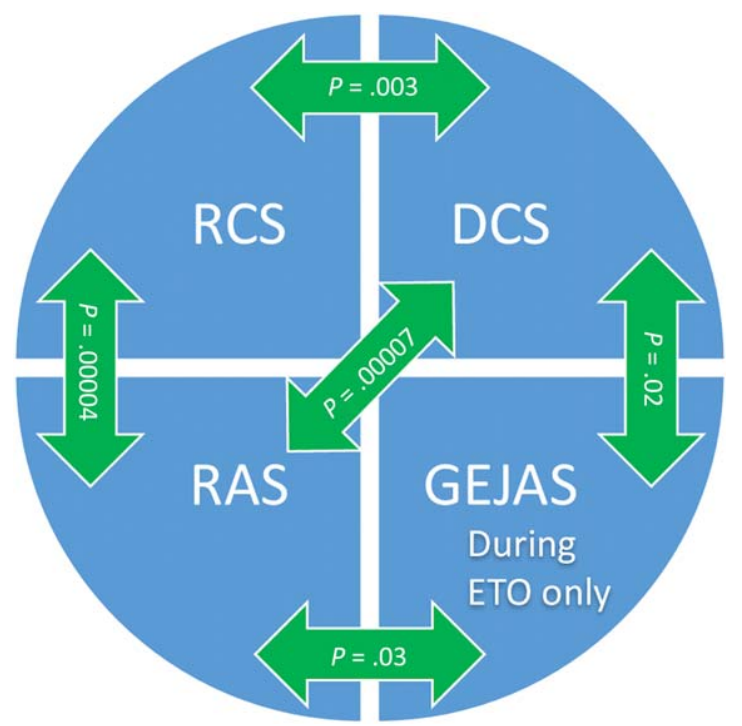

F I G URE 6 Correlations between scores. Correlations were identified between the 2 clinical scores (respiratory and digestive), the 2 respiratory scores (clinical and abnormalities), and between digestive clinical score and respiratory abnormalities score. The GEJ abnormalities score correlated with the digestive clinical score and with the respiratory abnormalities score only during ETO. DCS, digestive clinical score; ETO, temporary complete endotracheal tube obstruction; GEJAS, GEJ abnormalities score; RAS, respiratory abnormalities score; RCS, respiratory clinical score 
No gold standard has been established for endoscopic diagnosis of SHH in dogs. In the present study, SHH was diagnosed based on bulging and opening of the cardia, and visualization of the gastric mucosa $>2 \mathrm{~cm}$ cranial to the esophageal hiatus. This classification was derived from that used in man, where hiatal hernias can be typed according to the Makuuchi classification: grade A consists of a definite hiatal hernia (sac-shaped portion covered with gastric mucosa visible $\geq 3 \mathrm{~cm}$ above the hiatus); grade $\mathrm{B}$ is a minor hiatal hernia with gastric mucosa visible circumferentially $<3 \mathrm{~cm}$ above the hiatus; grade $\mathrm{C}$ is a minor hiatal hernia with gastric mucosa visible partially above the hiatus; and grade 0 consists of a normal status, with a mucosal junction visible below the hiatus. ${ }^{32}$ Based on our modified-Makuuchi classification, ETO allowed the scoring of 4 dogs as grade A (SHH), 12 as grade B or C (partial or complete eversion of the mucosa $<2 \mathrm{~cm}$ above the hiatus), and 4 dogs as grade 0 (no sliding of the GEJ) hiatal hernias. Overall, $80 \%$ of the dogs with BAOS in this study displayed variable degrees of SHH. This finding could explain the presence of digestive clinical signs in $65 \%$ of the same population of dogs with BAOS. Indeed, dogs with minor $\mathrm{HH}$ but without digestive clinical signs could include dogs with occult regurgitations, as described in man. ${ }^{33,34}$ Further studies using pH-metry or pressure manometry may help detect occult SHH and/or regurgitations in those dogs. ${ }^{22}$

Antiacid therapy is usually recommended in dogs with overt digestive clinical signs to treat upper esophageal, pharyngeal, and laryngeal inflammation secondary to regurgitation and/or vomiting. ${ }^{35-37}$ Systematic use of the scoring system and manipulations during endoscopic evaluation of the GEJ in dogs with BAOS could improve the detection of GEJ abnormalities and influence the therapeutic plan. Indeed, patients with only minor digestive clinical signs but with major GEJ abnormalities during manipulations may suffer from occult regurgitation and could benefit from antiacid therapy. Further studies are needed to assess the benefits of antiacid therapy in dogs with newly detected GEJ abnormalities after manipulations. In the author's experience, some dogs still present digestive clinical signs after corrective surgery of the obstructing upper airways and justify surgical correction of SHH. In this study, no dog required a surgical correction of the SHH (2-year follow-up). Evaluation of the GEJ under manipulations could potentially improve longitudinal monitoring of dogs, facilitate the diagnosis of persistent GEJ abnormalities, and help clinicians assess the need for surgical correction of the SHH.

The major limitation of this study is the lack of a control group comprising brachycephalic dogs without any respiratory and digestive abnormalities. Such a group would be difficult to identify, and the inclusion of dogs who underwent surgical correction of BAOS may provide an alternative. Five healthy Beagles were enrolled in a pilot study to evaluate the safety of the procedures but were not intended to serve as a control group. This sample size is too low to allow statistical analyses. Although very mild abnormalities of the GEJ (opening of the cardia) were observed in 2 of these dogs with MP, neither gastro-esophageal reflux nor gastric mucosal eversion were observed. While healthy dogs are not expected to face such transdiaphragmatic pressure gradient when awake, this observation highlights that manipulations could potentially create false positive GEJ abnormalities in healthy dogs. Further studies are needed to evaluate the effect of the manipulations on the dynamic of the GEJ in dogs that are free of BAOS. Additional groups of interest could include dogs suspected of SHH without BAOS and nonbrachycephalic dogs free of digestive and respiratory clinical signs.

\section{5 | CONCLUSIONS}

To our knowledge, this is the first prospective study reporting the effects of manipulations aimed at increasing transdiaphragmatic pressure gradient on the GEJ of dogs with BAOS. Our results highlight the limited diagnostic ability of standard radiography in detecting $\mathrm{SHH}$ and the potential of manipulations during endoscopy as a superior alternative. ETO was the only manipulation revealing a correlation among the digestive clinical signs, the GEJ abnormalities, and the respiratory abnormalities. ETO therefore appears to be the most effective manipulation to detect GEJ abnormalities during endoscopy of dogs with BAOS. The systematic use of manipulations during esophageal endoscopy could improve the diagnosis and understanding of SHH, and consequently improve the medical management of dogs with BAOS.

\section{CONFLICT OF INTEREST}

The authors declare no conflict of interest related to this report.

\section{REFERENCES}

[1] Gaskell CJ, Gibbs C, Pearson H. Sliding hiatus hernia with reflux oesophagitis in two dogs. J Small Anim Pract. 1974;15:503-509.

[2] Ellison GW, Lewis DD, Philips L, Tarvin GB. Esophageal hiatal hernia in small animals: literature review and a modified surgical technique. J Am Anim Hosp Assoc. 1987;23:391-399.

[3] Callan MB, Washabau RJ, Saunders HM, Kerr L, Prymak C, Holt D. Congenital esophageal hiatal hernia in the Chinese sharpei dog. J Vet Intern Med. 1993;7:210-215.

[4] White RN. A modified technique for surgical repair of esophageal hiatal herniation in the dog. J Small Anim Pract. 1993;34:599-603.

[5] Jergens AE. Diseases of the esophagus. In: Ettinger SJ, Feldman EC, eds. Textbook of Veterinary Internal Medicine. St. Louis, MO: Saunders; 2005:1298-1310.

[6] Poncet CM, Dupré GP, Freiche VG, Estrada MM, Poubanne YA, Bouvy BM. Prevalence of gastrointestinal tract abnormalities in 
73 brachycephalic dogs with upper respiratory syndrome. J Small Anim Pract. 2005;46:273-279.

[7] Bright RM, Sackman JE, Denovo C, Toal C. Hiatal hernia in the dog and cat: a retrospective study of 16 cases. J Small Anim Pract. 1990;31:244-250.

[8] Pratschke KM, Hughes JML, Skelly C, Bellenger CR. Hiatal herniation as a complication of chronic diaphragmatic herniation. J Small Anim Pract. 1998;39:33-38.

[9] Dieringer TM, Wolf AM. Esophageal hiatal hernia and megaesophagus complicating tetanus in two dogs. J Am Anim Hosp Assoc. 1991;199:87-89.

[10] Burnie AG, Simpson JW, Corcoran BM. Gastro-oesophageal reflux and hiatus hernia associated with laryngeal paralysis in a dog. J Small Anim Pract. 1989;30:414-416.

[11] Eliska O. Phreno-esophageal membrane and its role in the development of hiatal hernia. Acta Anat. 1973;86:137-150.

[12] Radmark T, Petterson GB. The contribution of the diaphragm and an intrinsic sphincter to the gastroesophageal antireflux barrier. An experimental study in the dog. Scand J Gastroenterol. 1989;24:85-94.

[13] Kahrilas PJ, Lin S, Chen J, Manka M. The effect of hiatus hernia on gastro-esophageal junction pressure. Gut. 1999;44:476-482.

[14] Sivacolundhu RK, Read RA, Marchevsky AM. Hiatal hernia controversies-a review of pathophysiology and treatment options. Aust Vet J. 2002;80:48-53.

[15] Pratschke KM, Fitzpatrick E, Campion D, McAllister H, Bellenger CR. Topography of the gastro-esophageal junction in the dog revisited: possible clinical implications. Res Vet Sci. 2004;76:171-177.

[16] Dupré G, Heidenreich D. Brachycephalic syndrome. Vet Clin North Am Small Anim Pract. 2016;46:691-707.

[17] Biancani P, Barwick K, Selling J, McCallum R. Effects of acute experimental esophagitis on mechanical properties of the lower esophageal sphincter. Gastroenterology. 1984;87:8-16.

[18] Liebermann-Meffert D, Klaus D, Vosmeer S, Allgöwer M. Effect of intraesophageal bile and acid $(\mathrm{HCl})$ perfusion on the action of the lower esophageal sphincter. Scand J Gastroenterol. Suppl. 1984;92:237-241.

[19] Hananoki M, Haruma K, Tsuga K, Hata J, Sumii K, Kajiyama G. Evaluation of lower esophageal sphincter pressure using endoscopic manometric sleeve assembly. J Gastroenterol Hepatol. 2000;15:121-126.

[20] Christensen J, Miftakhov R. Hiatus hernia: a review of evidence for its origin in esophageal longitudinal muscle dysfunction. Am J Med. 2000;108(4a):3S-7S.

[21] Han E. Diagnosis and management of reflux esophagitis. Clin Tech Small Anim Pract. 2003;18:231-238.

[22] Kahrilas PJ, Kim HC, Pandolfino JE. Approaches to the diagnosis and grading of hiatal hernia. Best Pract Res Clin Gastroenterol. 2008;22:601-616.

[23] Poncet CM, Dupré GP, Freiche VG, Bouvy BM. Long-term results of upper respiratory syndrome surgery and gastrointestinal tract medical treatment in 51 brachycephalic dogs. J Small Anim Pract. 2006;47:137-142.
[24] Tams TR. Diseases of the esophagus. In: Tams TR, ed. Handbook of Small Animal Gastroenterology. St. Louis, MO: Saunders; 2003:149-151.

[25] Bernaerts F, Talavera J, Leemans J, et al. Description of original endoscopic findings and respiratory functional assessment using barometric whole-body plethysmography in dogs suffering from brachycephalic airway obstruction syndrome. Vet J. 2010;183:95-102.

[26] Skinner DB. Hernias (hiatal, traumatic, and congenital). In: Berk JE, ed. Gastroenterology. Philadelphia, PA: W.B. Saunders; 1985:705-716.

[27] Hall JA, Magne ML, Twedt DC. Effect of acepromazine, diazepam, fentanyl-droperidol, and oxymorphone on gastresophageal sphincter pressure in healthy dogs. Am J Vet Res. 1987;48:556-557.

[28] Pratschke KM, Bellenger CR, McAllister H, Campion D. Barrier pressure at the gastroesophageal junction in anesthetized dogs. Am J Vet Res. 2001;62:1068-1072.

[29] Vandenbussche NL, Overeem S, Van Dijk JP, Simons PJ, Pevernagie DA. Assessment of respiratory effort during sleep: esophageal pressure versus noninvasive monitoring techniques. Sleep Med Rev. 2015;24:28-36.

[30] Roedler FS, Pohl S, Oechtering GU. How does severe brachycephaly affect dog's lives? Results of a structured preoperative owner questionnaire. Vet J. 2013;198:606-610.

[31] Haimel G, Dupré G. Brachycephalic airway syndrome: a comparative study between pugs and French bulldogs. J Small Anim Pract. 2015;56:714-719.

[32] Kusano M, Kouzu T, Kawano T, Ohara S. The prevalence of hiatus hernia in the Japanese. Gastroenterol Endosc. 2005;47: 962-973. In Japanese with English abstract.

[33] Blecker U, de Pont SM, Hauser B, Chouraqui JP, Gottrand F, Vandenplas Y. The role of "occult" gastroesophageal reflux in chronic pulmonary disease in children. Acta Gastroenterol Belg. 1995;58:348-352.

[34] Sarkar S, Thompson DG, Woolf CJ, Hobson AR, Millane T, Aziz Q. Patients with chest pain and occult gastroesophageal reflux demonstrate visceral pain hypersensitivity which may be partially responsive to acid suppression. Am J Gastroenterol. 2004;99:1998-2006.

[35] White DR, Heavner, SB, Hardy SM, Prazma J. Gastroesophageal reflux and Eustachian tube dysfunction in an animal model. Laryngoscope. 2002;112:955-961.

[36] Kook PH. Gastroesophageal reflux. In: Bonagura JD, Twedt DC, eds. Kirk's Current Veterinary Therapy XV. St. Louis, MO: Saunders; 2014:501-506.

[37] Bersenas AME. Antacid therapy. In: Bonagura JD, Twedt DC, eds. Kirk's Current Veterinary Therapy XV. St. Louis, MO: Saunders; 2014:505-508.

How to cite this article: Broux $\mathrm{O}$, Clercx $\mathrm{C}$, Etienne A-L, et al. Effects of manipulations to detect sliding hiatal hernia in dogs with brachycephalic airway obstructive syndrome. Veterinary Surgery. 2018;47:243-251. https://doi.org/10.1111/vsu.12735 